\title{
Deformation force model of indentation-flattening compound deformation technology
}

\author{
Zhongtang Wang ${ }^{1,}$, Lingyi Wang ${ }^{2, b}$ \\ ${ }^{1}$ School of Materials Science and Engineering, Shenyang Ligong University, Shenyang 110159, \\ China. \\ ${ }^{2}$ Shenyang Institute of Technology, Liaoning Fushun113122, China \\ aztwang@imr.ac.cn, blywang01@sina.cn
}

Keywords: AZ31 magnesium alloy, compound deformation, deformation force.

\begin{abstract}
It is discussed that the principle of indentation-flattening compound deformation (IFCDT) and parameter definition. The characteristics and stress state and strain state of IFCDT had been analyzed. Influence factors and influence laws on plastic deformation force had been analyzed. The calculation geometry model of mechanical parameters of IFCDT had been established. The model of deformation force of IFCDT had been given. The calculated results are in agreement with that of experimental, and the relative error is less than $19.4 \%$.
\end{abstract}

\section{Introduction}

Plastic deformation mechanics parameter is important parameter of making plastic deformation process and design of plastic forming tools. Therefore, the accurate calculation model of plastic deformation force parameter is an important basis for improving the precision plastic forming process and the quality of the product. Guo [1] had established the calculating model of tube extrusion force in the polar coordinate system. And the main stress method and power balance method is used to deduce the calculation formula of tube extrusion force, and the theoretical calculation results are agree well with the experimental results. Fu [2] had used the main stress method to establish predict model of rolling force and torque force during snake rolling. The influence of rolling parameters on rolling force and torque force had been analyzed, such as rate ratio, roll offset distance, reduction and friction coefficient. Liu [3] had established the calculation model of large diameter tube extrusion in polar coordinate system. The main stress method is used to determine the model of extrusion force of thick wall tube. And influence of extrusion ratio and extrusion angle on extrusion force had been analyzed. Mao [4] had used the stream function method to establish the mechanical model of the arbitrary curves die during extrusion process. Calculation method of tube extrusion force is obtained, and it is proved that the extrusion force calculation results are agreement with that of experiment. Ebrahimi [5] had used a new upper-bound approach to analyze the tube extrusion process, and a kinematical admissible velocity field is developed to evaluate the internal power and the power dissipated on frictional and velocity discontinuity surfaces.

\section{The characteristics IFCDT}

The principle of indentation-flattening compound deformation technology (IFCDT) is shown as Fig.1 (a). Intense tangential deformation can be produced by indentation deformation technology (referred to as first deformation), and it can increased the occurrence of dynamic recrystallization of magnesium alloy sheet. More twins and slip system can produce, and it is conducive to the grain refinement and the weakened of basal texture, and the formability of magnesium alloy sheet improved significantly. The magnesium alloy sheet which deformed by indentation technology is processed once again by flattening deformation (called second deformation). The dynamic recrystallization of magnesium alloy can be further aggravated, and grain is further refinement, and basal texture is further weakened. The performance and mechanical properties of magnesium alloy sheet improve 
significantly. Parameters of IFCDT defining as Fig.1(b), which are that deformation temperature (T), distance of waves $(s)$, reduction of IFCDT $(2 \Delta \mathrm{H})$, compress speed $(V)$, the coefficient of IFCDT $(\lambda)$, $\lambda=2 \Delta \mathrm{H} / s$, reduction ratio IFCDT $(\Phi) \Phi=2 \Delta \mathrm{H} / \mathrm{t}_{0}, t_{0}$ being thickness of initial blank.

(a)

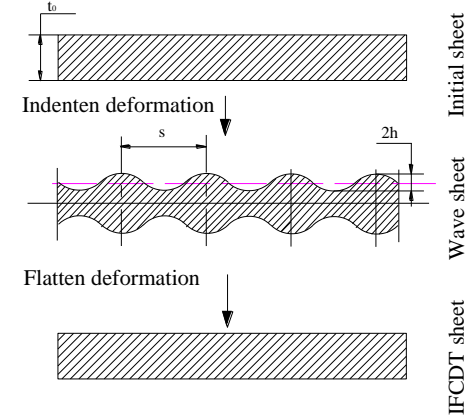

离

(b)

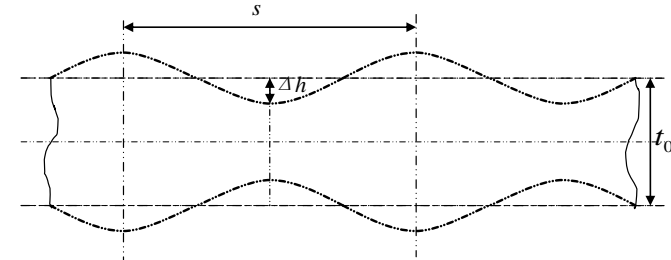

Fig.1 Principle of indentation-flat compound deformation (IFCDT) and parameter defining (a, principle of IFCDT; $b$, parameter defining.)

\section{Deformation force of indentation process}

The geometric model of indentation deformation is shown as Fig.2a. The principal stress method is used to solve the plastic deformation force.
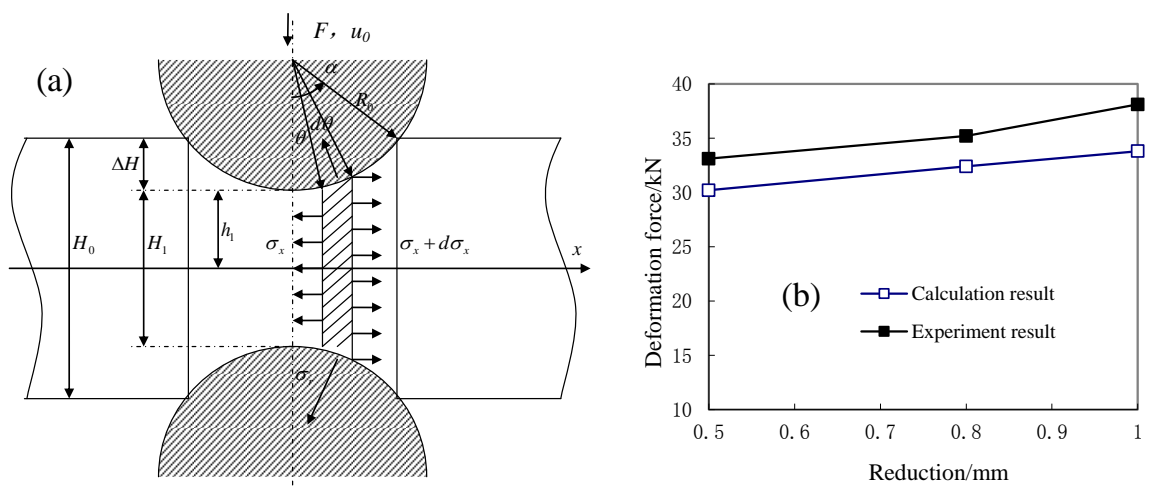

Fig.2 The geometry model of mechanical parameters of indentation deformation and calculated results (a, the geometric model of indentation deformation; $b$, results of calculation and experiment)

If friction force is neglecting, the equation of force balance of the micro-body is shown as equation (1).

$-\sigma_{x}\left[2 h_{1}+2 R_{0}(1-\cos \theta)\right] L+\left(\sigma_{x}+d \sigma_{x}\right)\left\{2 h_{1}+2 R_{0}[1-\cos (\theta+d \theta)]\right\} L-2 \sigma_{r} \cdot R_{0} d \theta \cdot \sin \left(\theta+\frac{d \theta}{2}\right) L=0$

According to the geometric model of indentation deformation, $H_{1}=2 h_{1}, \cos (\theta+d \theta) \approx \cos \theta, \sin$ $(\theta+d \theta / 2) \approx \sin \theta$, then equation (1) can be simplified to equation (2).

$$
d \sigma_{x}\left[h_{1}+R_{0}(1-\cos \theta)\right]-\sigma_{r} \cdot \mathrm{R}_{0} d \theta \cdot \sin \theta=0
$$

According to condition of yield criterion, $\sigma_{x}-\sigma_{r}=\sigma_{s}$, then $d \sigma_{x}=d \sigma_{r}$. A differential equation can be obtained, seen as equation (3).

$$
\frac{d \sigma_{r}}{\sigma_{r}}=\frac{R_{0} d \theta \cdot \sin \theta}{h_{1}+R_{0}(1-\cos \theta)}
$$

According to boundary condition, $\left.\sigma_{r}\right|_{\theta=\alpha}=-\sigma_{s}$, then, equation (4) and (5) can be obtained.

$$
\sigma_{r}=\frac{-\sigma_{s}\left[h_{1}+R_{0}(1-\cos \theta)\right]}{h_{1}+R_{0}(1-\cos \alpha)}
$$

Total deformation force of indentation process is given by equation (5).

$$
F_{1}=\int_{0}^{\alpha} \sigma_{r} R_{0} d \theta \cos \left(\theta+\frac{d \theta}{2}\right) L=\frac{\sigma_{s} R_{0} L}{h_{1}+R_{0}(1-\cos \alpha)}\left[\left(h_{1}+R_{0}\right) \sin \alpha-\frac{1}{2} R_{0} \alpha-\frac{1}{4} R_{0} \sin 2 \alpha\right]
$$

According to the geometric relationship, $h_{0}=h_{1}+R_{0}(1-\cos \alpha)$, then: 


$$
F_{1}=\frac{\sigma_{s} R_{0} L}{h_{0}}\left[\left(h_{0}+R_{0} \cos \alpha\right) \sin \alpha-\frac{1}{2} R_{0} \alpha-\frac{1}{4} R_{0} \sin 2 \alpha\right]
$$

If the convex tooth number is $\mathrm{n}$, then the total deformation force is given by equation (7).

$$
F_{1}=\frac{n \sigma_{s} R_{0} L}{h_{0}}\left[\left(h_{0}+R_{0} \cos \alpha\right) \sin \alpha-\frac{1}{2} R_{0} \alpha-\frac{1}{4} R_{0} \sin 2 \alpha\right]
$$

In which, $R_{0}$ is radius of convex tooth; $\alpha$ is press angle, $\cos \alpha=1-\left(H_{0}-H_{1}\right) / 2 R_{0}=1-\Delta H / R_{0}$; $\Delta H$ is reduction; $H_{1}$ is thickness of deformed sheet; $H_{1}=2 h_{1}, h_{1}=\left(H_{0}-2 \Delta H\right) / 2 ; H_{0}$ is thickness of initial sheet, $H_{0}=2 h_{0} ; n$ is convex tooth number; $\sigma_{s}$ is flow stress, which determined by equation (8)[6].

$$
\dot{\varepsilon}=5.718 \times 10^{20}\left[\sinh \left(0.0081 \sigma_{s}\right)\right]^{9.13} \exp (-252218 /(R T))
$$

In this example of AZ31 magnesium alloy sheet, the width of is $b=50$, and the thickness is $\mathrm{H}_{0}=7.0$, and radius of convex tooth $\mathrm{R}_{0}=4.0$, and reduction $\Delta \mathrm{H}$ is $0.5 \mathrm{~mm}, 0.8 \mathrm{~mm}, 1 \mathrm{~mm}$, respectively. Comparison between theoretical results and experimental results of indentation deformation force is shown as Fig.2b. The model results agree well with the experimental results, the relative error is less than $11.5 \%$. According to equation (7) and (8), it can be seen that the influence factors of composite deformation force include deformation temperature $(T)$, wave spacing $(\mathrm{S})$, reduction $(\mathrm{H})$, deformation velocity $(\mathrm{V})$, deformation ratio $\lambda=2 \mathrm{~h} / \mathrm{s}$.

\section{Calculation model of deformation force of flattening deformation}

Fig.3a is the geometry model of flattening deformation. According to principal stress method, the calculation formula of flattening deformation force can be obtained, seen equation (9).

$$
F_{2}=\frac{2 \mathrm{~K} b H_{1}}{\mu}\left(e^{\frac{\mu L}{H_{1}}}-1\right)
$$

In which, $\mathrm{K}$ is the maximum shear strength, $\mathrm{K}=0.5 \sigma_{\mathrm{s}}$; $\mathrm{L}$ is length of sheet, $\mathrm{L}=100 \mathrm{~mm}$; $\mathrm{b}$ is width of sheet, $\mathrm{b}=50 \mathrm{~mm} ; H_{1}$ is thickness of IFCDT sheet. In experiment, Value of $H_{1}$ is $6 \mathrm{~mm}, 5.4 \mathrm{~mm}, 5 \mathrm{~mm}$, respectively; $\mu$ is friction coefficient, $\mu=0.15$. Comparison between theoretical results and experimental results of flattening deformation is shown as Fig.3b, and the model results and experimental results. Well, the relative error is less than $19.4 \%$.

(a)

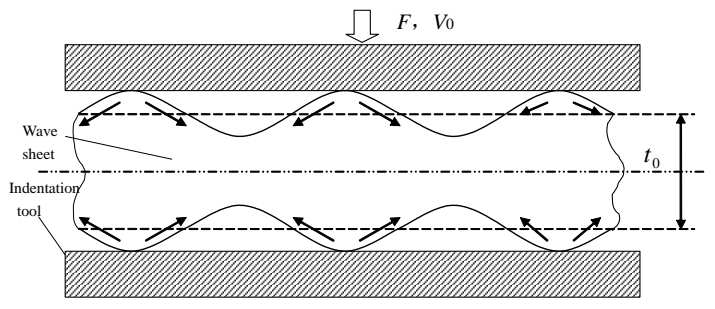

(a) The geometry model

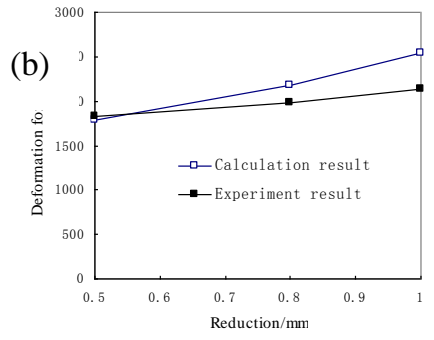

(b) Calculation results

Fig. 3 The geometry model of mechanical parameters of flattening deformation and calculated results (a, the geometry model; b, calculation results)

\section{Conclusion}

(1) Influence factors and influence laws on plastic deformation force had been analyzed. The calculation geometry model of mechanical parameters of IFCDT had been established.

(2) The model of deformation force of IFCDT had been given. The calculated results are in agreement with that of experimental, and his relative error is less than $19.4 \%$. 


\section{Acknowledgements}

This project is supported by National Natural Science Foundation of China (Grant No. 51575366)

\section{References}

[1]. GUO Sheng-li, LI De-fu, MA Zhi-xin, et al. Rediscussion on force of tube extrusion deduced by block method and the method of balance of work. Journal of plasticity engineering. Vol.15 (2008) No.1,p.31-35.

[2]. FU Yao, XIE Shui-sheng, XIONG Bai-qing, et al. Calculation of rolling force in snake rolling by slab method. Journal of plasticity engineering. Vol.17(2010)No.6, p.103-109.

[3]. LIU Qiang, FU Jianhua, JIA Lu, et al. Mechanical theory analysis of extrusion process for large diameter thick wall seamless tube. China Metal forming Equipment and Manufacturing Technology. Vol.50(2015)No.4, p.85-88.

[4]. MAO Yi-lun, ZHANG Qing-dong, ZHANG Xiao-feng, et al. Force of tube extrusion about different curved dies using basic flow function method. Engineering mechanics. Vol.31 (2014) No.2, p.210-213.

[5]. R.Ebrahimi, M.Reihanian, M.Kanaani, et al. An upper-bound analysis of the tube extrusion process. Journal of materials processing technology. Vol.199(2008), p.214-220.

[6]. WANG Zhong-tang, ZHANG Shi-hong, QI Guang-xia, et al. Constitutive equation of thermal deformation for AZ31 magnesium alloy. The Chinese Journal of Nonferrous Metals. Vol.18(2008) No.11, p.1977-1982. In Chinese. 